\title{
Recombinant lentivirus targeting the pleotrophin gene reduces pleotrophin protein expression in pancreatic cancer cells and inhibits neurite outgrowth of dorsal root ganglion neurons
}

\author{
JUN YAO, WEN-YAO LI, SHUO-GUO LI, XIAO-SHAN FENG and SHE-GAN GAO \\ Department of Oncology, First Affiliated Hospital, Henan University of Science and Technology, \\ Luoyang, Henan 471003, P.R. China
}

Received August 23, 2013; Accepted January 10, 2014

DOI: $10.3892 / \mathrm{mmr} .2014 .1918$

\begin{abstract}
The objectives of the present study were to construct the recombinant primate lentivirus-short hairpin RNA-pleiotrophin (pLV-shRNA-PTN) vector, to investigate the silencing effect of pLV-shRNA-PTN on PTN expression in MIA PaCa-2 cells and to observe the inhibition efficiency of pLV-shRNA-PTN on neurite outgrowth from dorsal root ganglion (DRG) neurons in vitro. The construction procedure for recombinant lentivirus pLV-shRNA-PTN has been described previously. In the present study, pLV-shRNA-PTN was used to infect MIA PaCa-2 pancreatic cancer cells and the efficiency of the knockdown of the PTN gene on day 7 following infection was analyzed using western blotting. The morphological changes in the cultured DRG neurons were observed by monoculture of DRG neurons and co-culture with MIA PaCa-2 cells in vitro. The recombinant lentivirus pLV-shRNA-PTN was successfully constructed. The western blot analysis showed that the inhibition rates of PTN expression were 46, 80, 20 and 21\%, respectively, following pLV-shRNA-PTN-A, $\mathrm{B}, \mathrm{C}$ and D infection. pLV-shRNA-PTN-B showed the highest knockdown efficiency. DRG neurons co-cultured with infected MIA PaCa- 2 cells were decreased in size when compared with the control, and there was a significant decrease in the number and length of neurites. The results suggest that efficient and specific knockdown of PTN in MIA PaCa-2 pancreatic cancer cells and the subsequent reduction in PTN expression results in the inhibition of neurite outgrowth from DRG neurons.
\end{abstract}

Correspondence to: Professor Xiao-Shan Feng, Department of Oncology, First Affiliated Hospital, Henan University of Science and Technology, 24 Jinhua Road, Luoyang, Henan 471003, P.R. China E-mail: yaojun74@163.com.cn

Key words: MIA PaCa-2 cells, pleiotrophin, RNA interference, neurite outgrowth, dorsal root ganglion neurons

\section{Introduction}

Pancreatic cancer is one of the most aggressive types of human malignancy with the lowest 5-year survival rate $(1,2)$. At the time of diagnosis, the majority of patients have locally advanced disease and/or distant metastatic lesions precluding radical operational resection $(3,4)$. Perineural invasion is considered to be an important factor of aggressive tumor behavior and is associated with local recurrence and poor outcome in patients with pancreatic cancer (5). Perineural invasion is defined as the presence of cancer cells within the epineural, perineural and endoneurial spaces of the neuronal sheet, and around nerves $(5,6)$. This infiltration results in severe pain and nerve damage (7). However it remains unknown why pancreatic cancer exhibits a high frequency of perineural invasion.

Pleiotrophin (PTN) is a type of neurotrophic factor, which is also known as a neurite growth-promoting factor. PTN can promote neurite outgrowth and neuronal survival in primarily cultured cortical neurons (8). PTN is mainly expressed during early embryogenesis. In adult tissues, PTN is markedly downregulated and is present only at minimal levels in certain tissues. It is not expressed in normal pancreatic tissues; however, it is expressed in pancreatic cancer cells (9). A high expression level of PTN has been observed in $78 \%$ of tumor samples from pancreatic cancer patients (10). PTN and $\mathrm{N}$-syndecan act as a receptor-ligand pair during the outgrowth of neurites. Anti-N-syndecan antibodies added to culture media exhibited an inhibitory effect on the PTN-induced outgrowth of neurites (11). In a previous study, we have confirmed that PTN and its receptor $\mathrm{N}$-syndecan can promote perineural invasion of pancreatic cancer cells (12). Therefore, PTN may be important in neurite outgrowth, thus promoting the perineural invasion of pancreatic cancer.

Chemically synthesized RNA interference (RNAi) molecules have been used to silence the expression of various genes in mammalian cell lines. The development of small interfering (si)RNAs to specifically inhibit gene expression by triggering RNAi pathways may be a potential strategy for the treatment of cancer (13). Recently, the development of more effective and stable gene nuclear silencing RNA-mediating systems has been of interest (14). In the present study, a lentiviral vector expressing short hairpin (sh)RNA against PTN was used to 
Table I. Sequences of shRNA/PTN clones.

shRNA/PTN clones Sequence

A

B

C

$\mathrm{D}$
CCGGGCAGCTGTGGATACTGCTGAACTCGAGTTCAGCAGTATCCACAGCTGC TTTTTG CCGGGCAACTGGAAGAAGCAATTTGCTCGAGCAAATTGCTTCTTCCAGTTGC TTTTTG CCGGGCCAGAAGACTGTCACCATCTCTCGAGAGATGGTGACAGTCTTCTGGC TTTTTG CCGGGCAAACCATGAAGACCCAGAGCTCGAGCTCTGGGTCTTCATGGTTTGC TTTTTG

shRNA, short hairpin RNA; PTN, pleiotrophin.

transduce MIA PaCa-2 cells. The silencing effect of primate lentivirus (pLV)-shRNA-PTN on the PTN gene in MIA PaCa-2 cells was investigated and the inhibition of neurite outgrowth from dorsal root ganglion (DRG) neurons in vitro was also examined.

\section{Materials and methods}

Chemicals and reagents. All chemicals were purchased from Sigma-Aldrich (St. Louis, MO, USA). Tris/glycine-buffered saline (TBS; 10X) was purchased from Bio-Rad (Hercules, CA, USA). Ethanol was obtained from IBI Scientific (Peosta, IA, USA). Monoclonal mouse anti-human PTN, anti-glyceraldehyde-3-phosphate dehydrogenase (GAPDH) antibodies and peroxidase-coupled goat anti-mouse $\mathrm{IgG}$ secondary antibody were purchased from Santa Cruz Biotechnology, Inc. (Santa Cruz, CA, USA).

Design of shRNA-PTN and construction of pLV-shRNA-PTN. Four lentiviral transduction particles encoding shRNA against PTN (shRNA/PTN) were purchased from Sigma-Aldrich. The lentiviral shRNA vector map is shown in Fig. 1. Four 21-nucleotide shRNA duplexes from different sections of human PTN mRNA (GenBank accession no. NM002825) were designed using the MISSION search database (www. sigma-aldrich.com/missionsearch), which is produced and distributed under license by the Massachusetts Institute of Technology (Cambridge, MA, USA). The shRNA sequences analyzed are listed in Table I.

Transduction of $p L V$-shRNA-PTN into MIA PaCa-2 cells. The MIA PaCa-2 cell line, collected from a poorly differentiated human pancreatic adenocarcinoma, was transduced with pLV-shRNA-PTN. The cells were plated at a density of $5 \times 10^{6}$ cells/well and transduced with $1 \mu \mathrm{g} \mathrm{pLV-shRNA-PTN}$ (multiplicity of infection, 1) in 96-well tissue culture plates. As a control, MIA PaCa-2 cells were also transduced with the same quantity of viral vector containing noncoding shRNA (Sigma-Aldrich). The transduced cells were selected in cell culture medium containing $10 \mu \mathrm{g} / \mathrm{ml}$ puromycin. Visible clones were picked from 96-well plates, expanded in 24-well tissue culture plates and finally transferred to regular cell culture flasks. All the clones were screened using molecular biology tools, using the following stages: i) cell selection and pre-treatment; ii) cell disruption; iii) extraction; iv) purification (including salting out, organic solvent precipitation, organic solvent extraction, ultracentrifugation and crystallization); and v) condensation and preservation. The expression of PTN protein was determined by western blotting using the PTN-specific antibody.

Western blotting analysis of PTN protein. Monolayer MIA PaCa-2 cells were grown to confluence in 90\% Dulbecco's modified Eagle's medium (DMEM) with $10 \%$ fetal bovine serum (FBS). Upon reaching confluence, the medium was removed and the monolayer cells were washed with cold phosphate-buffered saline and lysed in ice-cold lysis buffer (Santa Cruz Biotechnology, Inc.) for the radioimmunoprecipitation assay. Protein isolation was followed according to the manufacturer's instructions. The concentration of protein was measured according to the manufacturer's instructions of the Quant-it Protein Assay kit (Invitrogen Life Technologies, Carlsbad, CA, USA) using the Qubit fluorometer (Invitrogen Life Technologies). For protein expression analysis, $45 \mu \mathrm{g}$ protein was separated on 4-20\% SDS-polyacrylamide gels. Following electrophoresis, the proteins were transferred to polyvinylidene difluoride membranes following Bio-Rad instructions. After the proteins were transferred, the membranes were blocked for $1 \mathrm{~h}$ using $0.2 \%$ I-block solution (Applied Biosystems, Foster City, CA, USA) in TBS containing 0.05\% Tween-20. The blocked membranes were probed sequentially with anti-PTN and anti-GAPDH antibodies, followed by incubation with the secondary antibodies. Unbound secondary antibody was removed by washing the membrane three times with TBS containing $0.05 \%$ Tween-20. The bands were visualized using an enhanced chemiluminescence system (UVP, Upland, CA, USA).

Separation and culture of DRG neurons. Newborn mice were euthanized with carbon dioxide and sterilized using $75 \%$ ethanol. The study was approved by the Ethics Committee of First Affiliated Hospital, Henan University of Science and Technology (Luoyang, China). The spines were cut open and the DRG neurons were isolated from the vertebrae. The spinal cords with the attached DRG neurons were removed from the vertebrae. DRG neurons from the cervical and thoracic areas were dissected under sterile conditions and were transferred to a $15 \mathrm{ml}$ tube with $5 \mathrm{ml}$ D-Hanks' solution then centrifuged at $70 \mathrm{x}$ g for $5 \mathrm{~min}$. The supernatant was removed and $20 \mu \mathrm{l}$ of $2.5 \%$ trypsin was added to the tube, which was incubated for $20-25 \mathrm{~min}$ at $37^{\circ} \mathrm{C}$, followed by centrifugation at $800 \mathrm{rpm}$ for $5 \mathrm{~min}$. The supernatant was removed and $2 \mathrm{ml}$ serum-supplemented medium (DMEM/F12; Gibco-BRL, Grand Island, NY, USA) containing 10\% heat-inactivated FBS (Sigma, St. Louis, 


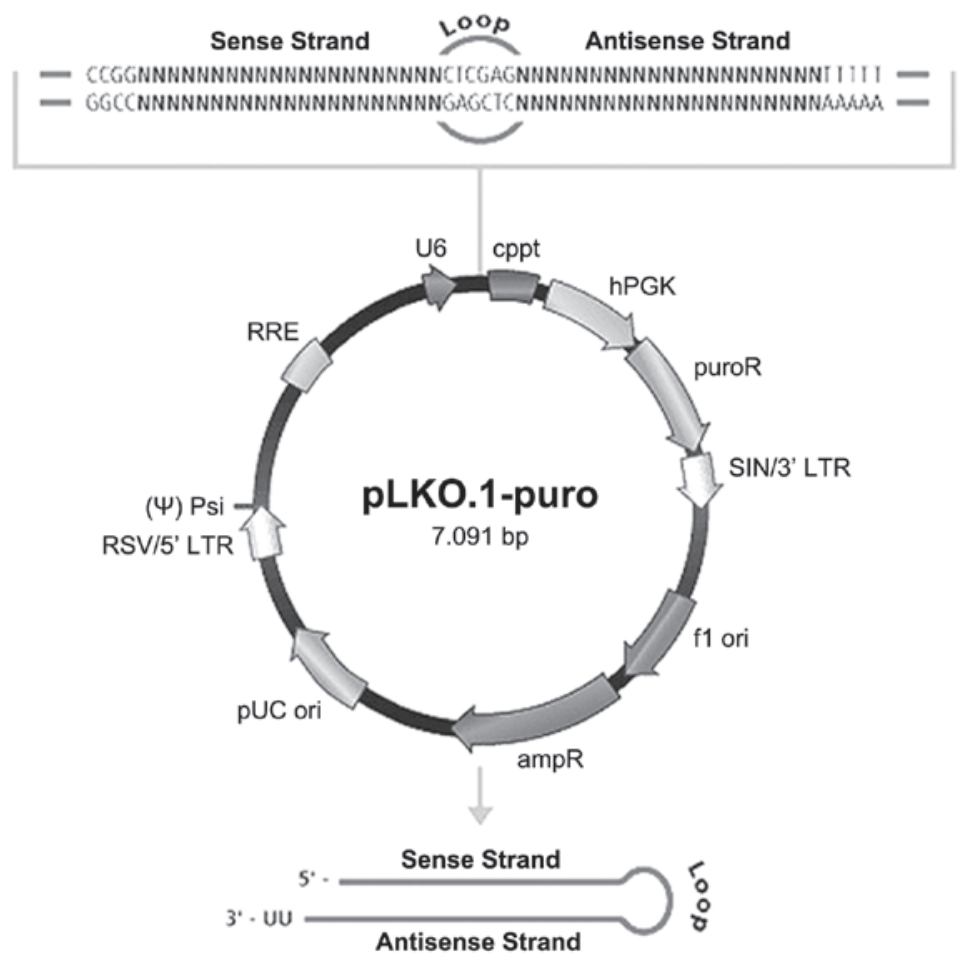

Figure 1. Physical map of lentiviral short hairpin (sh)RNA transfer vector constructs (obtained from Sigma-Aldrich, St. Louis, MO, USA). Pleiotrophin sequences are cloned into the pLKO.1-puro vector. In this vector, $\mathrm{U}_{6}$ is a robust pol III promoter with precise initiation and termination of shRNA transcription.

MO, USA) was added to the tube and mixed. The neurons were counted and seeded in 6-well culture plates at $10^{5}$ cells per well.

Cell culture. The MIA PaCa-2 cell line was obtained from the American Type Culture Collection (Manassas, VA, USA). The model was established by co-culturing DRG neurons with MIA PaCa-2 cells as previously described (15). Dissociated DRG neurons were seeded on coverglasses treated with polylysine (10\%, Sigma-Aldrich) and placed in 12-well culture plates. A metal rack was placed in each well after the cells had adhered to the coverglasses. The MIA PaCa- 2 cells were also seeded onto coverglasses, which were placed in 12-well plates, and $2 \mathrm{ml}$ DMEM/F12 medium with $10 \%$ FCS was added into each well. On the day of infection, the previous culture medium was removed from the cells, $50 \mu 1$ recombinant pLV-shRNA-PTN and $2 \mathrm{ml}$ fresh DMEM/F12 medium containing 2\% FCS (at an MOI of 50) were added to the infection group, and $2 \mathrm{ml}$ fresh DMEM/F12 medium containing 2\% FCS without lentiviral stock was added to the control (uninfected) group. Following incubation at $37^{\circ} \mathrm{C}$ for $24 \mathrm{~h}$, the coverglasses were placed on the metal racks. The MIA $\mathrm{PaCa}-2 / \mathrm{DRG}$ neurons were routinely cultured in a serum-supplemented medium at $37^{\circ} \mathrm{C}$ (DMEM/F12; GIBCO) containing 10\% heat-inactivated FBS in a humidified atmosphere of $95 \% \mathrm{O}_{2}$ and $5 \% \mathrm{CO}_{2}$. DRG neurons were also cultured alone as a control.

Image analysis. Following cell culture for 1, 3, 5 and 7 days, the cell growth was observed, and the cyton size and axon length were photographed by an inverted imaging microscope at x200 magnification (Nikon, Tokyo, Japan). Ten random visual fields in the infected and control groups were observed and analyzed using Motic software (Macao, China). Neurite outgrowth was evaluated by measuring the vertical length between the spots where the neurite grew from the pericaryon and its terminus. The major and minor axis were denoted as a and b, respectively. The diameter of the cell body was measured by multiplying $\mathrm{a}$ and $\mathrm{b}$ and extracting its square root. All units are presented in micrometers.

Statistical analysis. The images obtained from western blotting were scanned and analyzed using the Quantity One software (Bio-Rad). The ratios of PTN to GAPDH were calculated. The statistical significance between the control and infected groups was calculated using one-way analysis of variance, and $\mathrm{P}<0.05$ was considered to indicate a statistically significant difference.

\section{Results}

Suppression of PTN protein expression by recombinant lentivirus $p L V$-shRNA-PTN. The PLV-shRNA-PTN vectors were successfully constructed with the viral titer up to $4.4 \times 10^{8} \mathrm{TU} / \mathrm{ml}$. Western blot analysis using anti-PTN-specific antibodies on day 7 of culture revealed that PTN protein expression in MIA PaCa-2 cells infected with pLV-shRNA-PTN was suppressed when compared with the control. The inhibition rates of PTN protein expression following pLV-shRNA-PTN-A, -B, $-\mathrm{C}$ and $-\mathrm{D}$ infection were 46, 80, 20 and 21\%, respectively, (Fig. 2). pLV-shRNA-PTN-B exhibited the highest knockdown efficiency. These results indicate that although all pLV-shRNA-PTN plasmids inhibited the production of PTN protein in MIA PaCa-2 cells, pLV-shRNA-PTN-B was the more potent inhibitor for PTN protein expression in MIA PaCa-2 cells. In follow-up experiments, the pLV-shRNA-PTN-B plasmid was used in the experimental condition groups. 


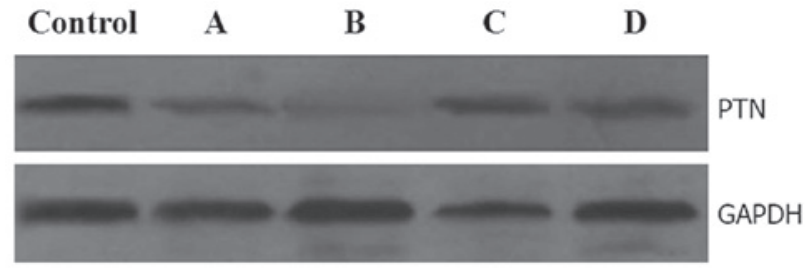

Figure 2. Inhibitory effect of recombinant pLV-shRNA-PTN on PTN protein expression in the MIA PaCa-2 pancreatic cancer cell line. pLV, primate lentivirus; shRNA, short hairpin RNA; PTN, Pleiotrophin; GAPDH, glyceraldehyde 3-phosphate dehydrogenase.
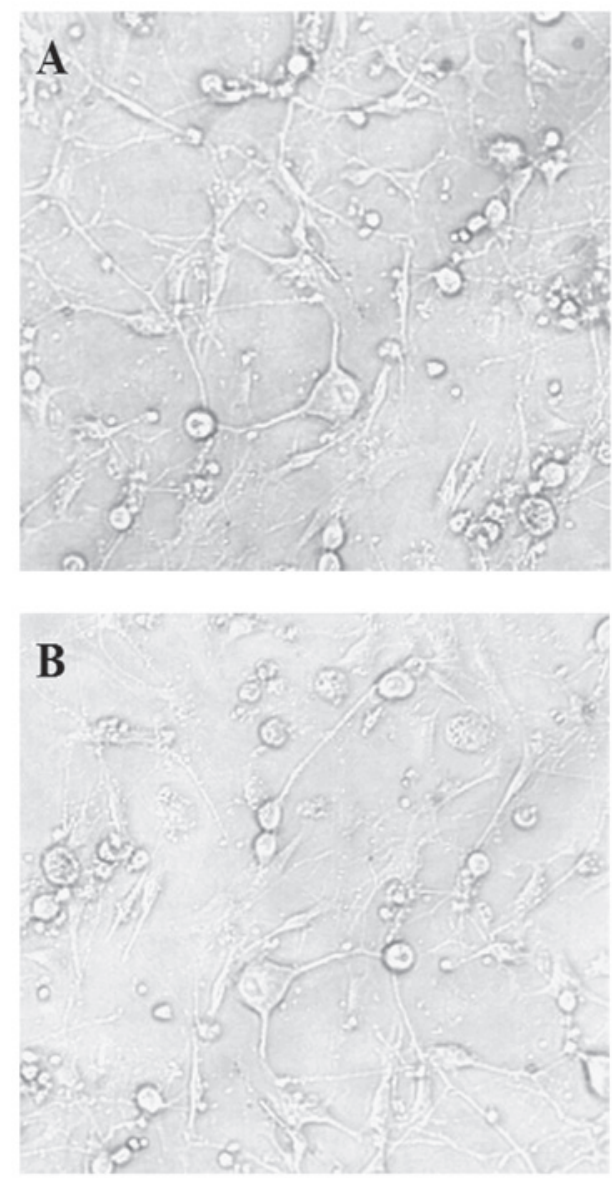

Figure 3. In the control group of dorsal root ganglion neurons co-cultured with MIA PaCa- 2 cells, cancellous association emerged and the neurites passed through one axon to another and formed a network (A). In the infected group co-culture, the neurites were small and indistinct, and there was no cancellous association (B). The co-cultures were observed at $\mathrm{x} 400$ magnification.

Dynamic observation of neuron growth. On day 1 of monoculture (cells grown alone), the cells were large, lucent and smooth, and growth cones around the cell bodies were observed. During the cell culture, no obvious morphological changes in the DRG neurons were identified. On day 7, the number of monocultured neurons decreased and a few neurons exhibited extended neurites. In the co-cultures of DRG neurons and MIA PaCa-2 cells, the cytons gradually became fusiform. On day 7 , the neurites in the control group had emerged and increased in length when compared with the group infected with pLV-shRNA-PTN. The length of neurites in the control group progressively increased with time and a cancellous

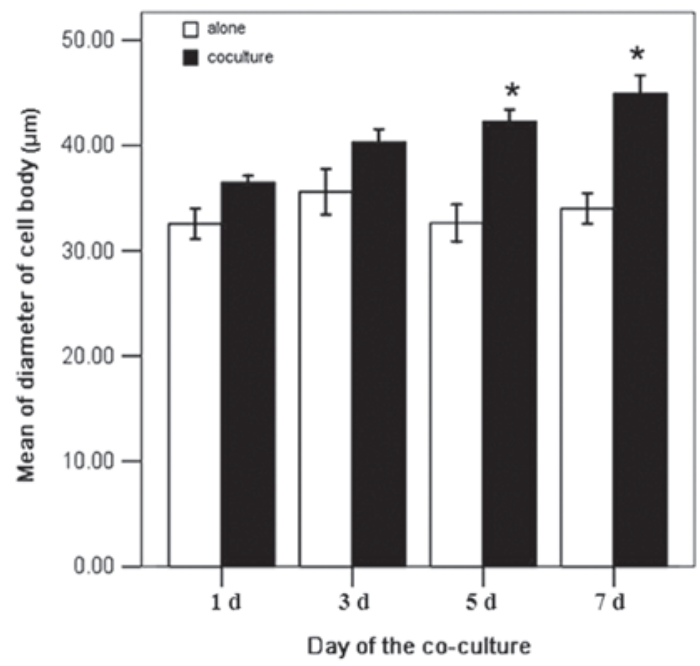

Figure 4. The diameters of the dorsal root ganglion (DRG) cell bodies in the two groups were measured on days 1, 3, 5 and 7 of co-culture with MIA $\mathrm{PaCa}-2$ cells. On days 1 and 3 , there was no significant difference identified in the diameters of the cell bodies between the groups. On days 5 and 7, the neurons in the control group were revealed to be significantly larger than those in the infected group. ${ }^{*} \mathrm{P}<0.05$ compared with control.

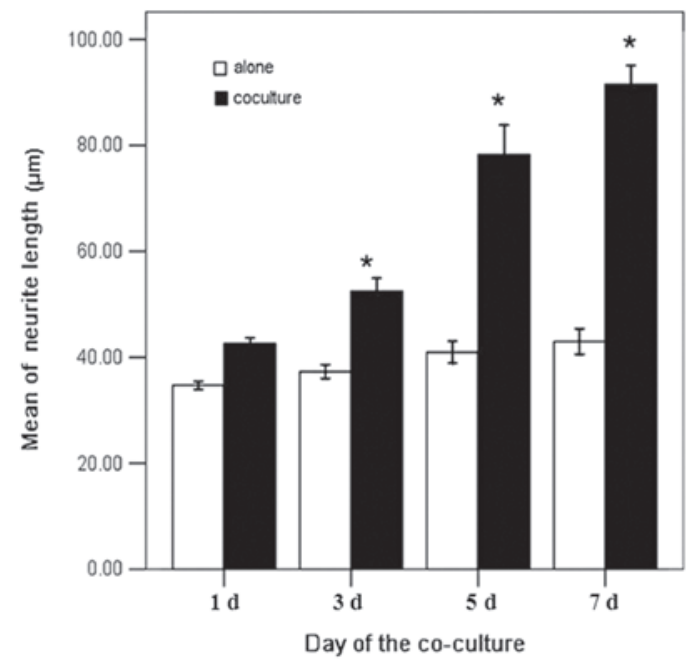

Figure 5. The neurite outgrowths of the two groups of dorsal root ganglion (DRG) cells were measured on days 1, 3, 5 and 7 of co-culture with MIA PaCa-2 cells. In the control group (black bar chart), the number of neurite outgrowths was significantly greater and axons grew quicker than those of the infected group (white bar chart) on days 5 and 7. The neurite outgrowth in the infected group was slow and no significant differences were identified in the lengths of neurites between different days. " $\mathrm{P}<0.05$.

association among neurites emerged (Fig. 3A), which was not observed in the infected group (Fig. 3B). The cells in thetwo groups schizolysed markedly and the cell envelopes were not smooth.

Measurement of neuron cyton size and neurite outgrowth. The diameters of the cell bodies from the two groups were measured on days $1,3,5$ and 7 . In the control group, the diameters of the cell bodies on these co-culture days were $35.7,39.0,41.1$ and $43.0 \mathrm{~mm}$, respectively. In the infected group, the diameters of outgrown neurites on the same days were $31.2,34.9,31.6$ and $32.1 \mathrm{~mm}$, respectively. The diameters of the cell bodies in the control group were 1.14, 1.13, 1.30, 
and 1.34 times larger than those in the infected group with a significant difference $(\mathrm{P}<0.05$, Fig. 4). The neurite outgrowths of the two groups were also measured on days $1,3,5$, and 7 . In the control group, the neurite outgrowths were 43.2, 52.5, 79.6, and $89.3 \mathrm{~mm}$, respectively. In the infected group, the neurite outgrowths were $33.7,35.1,40.8$, and $42.4 \mathrm{~mm}$, respectively. The neurite outgrowths of the control group were 1.28, 1.50, 1.95 and 2.11 times larger than in the infected group with a significant difference ( $\mathrm{P}<0.05$, Fig. 5).

\section{Discussion}

Perineural invasion is defined as the presence of cancer cells along nerves and/or within the epineurial, perineurial, and endoneurial spaces of the neuronal sheath (6). Perineural invasion is a multifactorial process involving various signaling molecules from different signaling pathways $(5,16,17)$. These signaling molecules include nerve growth factors, neurotrophic factors, chemokines, and cell-surface ligands and receptors $(18,19)$. Pancreatic ductal adenocarcinomas cells exhibit marked neurotrophic effects and the pancreas is in close proximity to several neural plexuses, which may partially explain the high incidence of perineural invasion in pancreatic cancer (20).

RNAi-triggered mRNA destruction is widely used for the modulation of gene expression. This technique is under extensive investigation and has been recently applied to the inhibition of PTN (21). In the present study, pLV-shRNA-PTN was used to establish an MIA PaCa-2 cell line with stable suppression of the PTN gene. It was confirmed that transduction by shRNA/PTN lentiviral vector constructs produced a pronounced PTN gene-silencing at the protein level. However, not all positive synthetic shRNA oligos can exhibit effective functions in vector-based RNAi (22). The silencing may depend on the transduction efficiency and the half-life of shRNA/PTN. The accuracy with which the efficiency of an individual siRNA can be predicted is also unclear (23). The results of the present study confirm that not all shRNAs were capable of knocking down PTN expression to the same extent when the shRNAs were introduced into cells. Therefore, shRNAs exhibit variable capabilities in mediating RNAi knockdown. The pLV-shRNA-PTN knockdown cells were subsequently examined, and Clone B exhibited the highest knockdown efficiency on protein expression (80\% downregulation when compared with the vector control).

The previous mechanisms regarding perineural invasion have demonstrated that cancer cells exhibit an initiative effect whilst nerve fibers exhibit a passive effect. However, whether cancer cells can promote the functions of nerve cells and nerve fiber effects in the perineural invasion of pancreatic cancer has not been widely reported, as the potential mechanisms are not clear. The activity of neurons can be evaluated by observing neurite outgrowth and cyton size. In the in vitro model system used in the present study, enhanced cyton size, neurite outgrowth and neuron activity were observed in the presence of MIA $\mathrm{PaCa}-2$ cells from the control group, supporting the hypothesis that cancer cells can promote the functions of nerve cells. These results indicate that cancer cells also have tropism towards the growth of nerve cells. This effect may be involved in mediating the invasion of cancer cells along nerves. In the control group, the neurites grew significantly on days 5-7 of the culture, suggesting that certain factors mediate the growth of neurites. It was hypothesized that specific growth factors produced by nerve cells or cancer cells may promote the growth and survival of nerve cells, and exhibit neurotropism. As a neurite growth-promoting factor, PTN can synergistically promote the development of perineural invasion in pancreatic cancer.

PTN is also involved in the regeneration of peripheral nerves following nerve injury and functional recovery following neural transplantation in rats $(24,25)$. The available evidence suggests that PTN bound to $\mathrm{N}$-syndecan promotes neurite outgrowth from DRG neurons. In the present study, DRG neurons were selected due to their involvement in the perineural invasion of pancreatic cancer (26). DRG neurons were dissociated from newborn mice for monoculture or co-culture with MIA PaCa-2 cells. The results indicate that pLV-shRNA-PTN can be used to infect MIA PaCa-2 cells and suppress PTN gene expression efficiently. Compared with the number of control cells, the number of DRG neurons co-cultured with infected MIA PaCa-2 cells and the number of extended neurites was significantly decreased. These results are concurrent with those of our previous study (21).

In conclusion, the lentiviral construct pLV-shRNA-PTN can efficiently and specifically knockdown PTN in the MIA $\mathrm{PaCa}-2$ pancreatic cancer cell line, and also inhibit the neurite outgrowth from DRG neurons in vitro. Therefore, the PTN gene and pLV-shRNA-PTN are important for investigating perineural invasion in pancreatic cancer. We will establish a model of pancreatic cancer in situ in nude mice in future studies. By injecting pLV-shRNA-PTN plasmid, the effects of PTN gene and lentiviral construct pLV-shRNA-PTN on perineural invasion of pancreatic cancer may be further investigated.

\section{Acknowledgements}

This study was supported by the National Natural Science Foundation of China (grant no. U1204819) and by the Health Science and Technology Innovation Talents Program of Henan Province (grant no. 4203).

\section{References}

1. Siegel R, Naishadham D and Jemal A: Cancer statistics, 2012. CA Cancer J Clin 62: 10-29, 2012.

2. Mössner J: What's new in therapy of pancreatic cancer. Dig Dis 28: 679-683, 2010

3. Han SL, Zhang WJ, Zheng XF, Shen X, Zeng QQ and Ke QH: Radical resection and outcome for malignant tumors of the pancreatic body and tail. World J Gastroenterol 15: 5346-5351, 2009.

4. Chiang KC, Yeh CN, Lee WC, Jan YY and Hwang TL: Prognostic analysis of patients with pancreatic head adenocarcinoma less than $2 \mathrm{~cm}$ undergoing resection. World J Gastroenterol 15: 4305-4310, 2009.

5. Bapat AA, Hostetter G, Von Hoff DD and Han H: Perineural invasion and associated pain in pancreatic cancer. Nat Rev Cancer 11: 695-707, 2011.

6. Liebig C, Ayala G, Wilks JA, Berger DH and Albo D: Perineural invasion in cancer: a review of the literature. Cancer 115: 3379-3391, 2009.

7. Ceyhan GO, Michalski CW, Demir IE, Müller MW and Friess H: Pancreatic pain. Best Pract Res Clin Gastroenterol 22: 31-44, 2008.

8. Takamatsu H, Itoh M, Kimura M, Gospodarowicz D and Amann E: Expression and purification of biologically active human OSF-1 in Escherichia coli. Biochem Biophys Res Commun 185: 224-230, 1992. 
9. Weber D, Klomp HJ, Czubayko F, Wellstein A and Juhl H: Pleiotrophin can be rate-limiting for pancreatic cancer cell growth. Cancer Res 60: 5284-5288, 2000.

10. Souttou B, Juhl H, Hackenbruck J, et al: Relationship between serum concentrations of the growth factor pleiotrophin and pleiotrophinpositive tumors. J Natl Cancer Inst 90: 1468-1473, 1998.

11. Raulo E, Chernousov MA, Carey DJ, Nolo R and Rauvala H: Isolation of a neuronal cell surface receptor of heparin binding growth-associated molecule (HB-GAM). Identification as N-syndecan (syndecan-3). J Biol Chem 269: 12999-13004, 1994.

12. Yao J, Ma Q, Wang L and Zhang M: Pleiotrophin expression in human pancreatic cancer and its correlation with clinicopathological features, perineural invasion, and prognosis. Dig Dis Sci 54: 895-901, 2009.

13. Lage H: Potential applications of RNA interference technology in the treatment of cancer. Future Oncol 1: 103-113, 2005.

14. Song J, Cao L and Li Y: RNA interference-mediated inhibition of survivin and VEGF in pancreatic cancer cells in vitro. Mol Med Rep 7: 1651-1655, 2013

15. Fukuda J: Nerve cells of adult and aged mice grown in a monolayer culture: age-associated changes in morphological and physiological properties of dorsal root ganglion cells in vitro. Dev Neurosci 7: 374-394, 1985.

16. Ceyhan GO, Demir IE, Altintas B, et al: Neural invasion in pancreatic cancer: a mutual tropism between neurons and cancer cells. Biochem Biophys Res Commun 374: 442-447, 2008.

17. Gil Z, Cavel O, Kelly K, et al: Paracrine regulation of pancreatic cancer cell invasion by peripheral nerves. J Natl Cancer Inst 102: $107-118,2010$

18. Ma J, Jiang Y, Jiang Y, Sun Y and Zhao X: Expression of nerve growth factor and tyrosine kinase receptor $\mathrm{A}$ and correlation with perineural invasion in pancreatic cancer. J Gastroenterol Hepatol 23: 1852-1859, 2008
19. Marchesi F, Piemonti L, Fedele G, et al: The chemokine receptor CX3CR 1 is involved in the neural tropism and malignant behavior of pancreatic ductal adenocarcinoma. Cancer Res 68: 9060-9069, 2008.

20. Ceyhan GO, Schäfer KH, Kerscher AG, et al: Nerve growth factor and artemin are paracrine mediators of pancreatic neuropathy in pancreatic adenocarcinoma. Ann Surg 251: 923-931, 2010.

21. Yao J, Zhang M, Ma QY, Wang Z, Wang LC and Zhang D PAd-shRNA-PTN reduces pleiotrophin of pancreatic cancer cells and inhibits neurite outgrowth of DRG. World J Gastroenterol 17: 2667-2673, 2011.

22. Celius T, Garberg P and Lundgren B: Stable suppression of MDR1 gene expression and function by RNAi in Caco-2 cells. Biochem Biophys Res Commun 324: 365-371, 2004.

23. Schuck S, Manninen A, Honsho M, Füllekrug J and Simons K: Generation of single and double knockdowns in polarized epithelial cells by retrovirus-mediated RNA interference. Proc Natl Acad Sci USA 101: 4912-4917, 2004.

24. Blondet B, Carpentier G, Lafdil F and Courty J: Pleiotrophin cellular localization in nerve regeneration after peripheral nerve injury. J Histochem Cytochem 53: 971-977, 2005.

25. Hida H, Masuda T, Sato T, Kim TS, Misumi S and Nishino H: Pleiotrophin promotes functional recovery after neural transplantation in rats. Neuroreport 18: 179-183, 2007.

26. Dai H, Li R, Wheeler T, et al: Enhanced survival in perineural invasion of pancreatic cancer: an in vitro approach. Hum Pathol 38: 299-307, 2007. 